\title{
Spatio-temporal analysis of fox rabies cases in Germany 2005-2006
}

\author{
Matthias Eckardt,, ${ }^{1,2}$ Conrad Freuling, ${ }^{3}$ Thomas Müller, ${ }^{3}$ Thomas Selhorst ${ }^{1}$ \\ ${ }^{1}$ Unit Epidemiology, Statistics and Mathematical Modelling, Federal Institute for Risk Assessment, \\ Berlin; ' Department of Computer Science, Humboldt-Universität zu Berlin, Berlin; 3Institute of \\ Molecular Virology and Cell Biology, Friedrich-Loeffler-Institut, Federal Research Institute for \\ Animal Health, Riems, Germany
}

\begin{abstract}
Aiming to achieve new insights into rabies dynamics, this paper is the first to investigate fox rabies in Germany from a space-time pattern perspective. Based on a locally restricted dataset covering a fourteen month period, our findings indicate a strongly aggregated spatiotemporal point pattern resulting from an inhomogeneous stochastic process. In contrast to spatial or temporal approaches or cellular automata, our analysis focuses on the disease dynamics in time and space in a continuous time domain. Our findings confirm existing theories regarding fox rabies control highlighting the potential risk of urban areas and the need for effective rabies vaccination.
\end{abstract}

\section{Introduction}

This paper investigates the incidence of fox rabies from a spatiotemporal point process perspective aiming to contribute new insights into the disease dynamics. More formally, we assume that the observed incident cases are realizations from a stochastic process evolving in a spatio-temporal domain. Thus, our analysis is based on a random

Correspondence: Matthias Eckardt, Unit Epidemiology, Statistics and Mathematical Modelling, Federal Institute for Risk Assessment, 10609 Berlin, Germany

Tel: +49.301 .84121969 - Fax: +49.301 .84122961$

E-mail: Matthias.Eckardt@bfr.bund.de

Key words: Spatio-temporal point data; Spatio-temporal disease pattern, Clustered process, Germany.

Received for publication: 12 January 2015.

Revision received: 11 February 2015.

Accepted for publication: 25 February 2015.

(c) Copyright M. Eckardt et al., 2015

Licensee PAGEPress, Italy

Geospatial Health 2015; 10:313

doi:10.4081/gh.2015.313

This article is distributed under the terms of the Creative Commons Attribution Noncommercial License (by-nc 3.0) which permits any noncommercial use, distribution, and reproduction in any medium, provided the original author(s) and source are credited. countable set of events:

$$
\left\{\left(s_{i}, t_{i}\right), i=1, \ldots, k\right\}
$$

where $s_{i} \in \mathrm{S}$ refers to the location and $t_{i} \in \mathrm{T}$ to the time of occurrence of event $i$ within a bounded spatio-temporal observation window $\mathrm{S} \times \mathrm{T}$ $\subset \mathbb{R} 2 \times \mathbb{R}$ (cf. Diggle, 2014; Cressie and Wikle, 2011).

Rabies is present worldwide, but the greatest burden of the disease is in the developing countries, where dog-mediated rabies causes approximately 74,000 [95\% confidence interval (CI) 48,000-106,000] human deaths annually, in particular in Asia and Africa. Tragically, $40 \%$ of people who are bitten by suspect rabid animals are children (World Health Organization, 2013).

In Europe, where rabies is largely mediated by wildlife carnivores, oral rabies vaccination (ORV) is a proven efficient method of control. During the past decades, the implementation of ORV considerably improved the rabies situation in Europe and several countries have been officially recognized as being free of terrestrial rabies (Freuling et al., 2013). In Germany, fox rabies has been present since the 1950 s. The application of large scale ORV reduced the endemic area to a minimum, i.e. to a limited part of the South of the Federal State of Hessen. In 2005, the final phase of terrestrial rabies eradication in Germany was put at risk by a severe setback due to re-introduction of the disease in the state of Rhineland-Palatinate from neighbouring Hessen after seven years of absence.

In this paper, we restrict our analysis to a 14-month period in the area on the Western side of the River Rhine in Rhineland-Palatinate. This outbreak lasted for one year only due to swift reactions and application of an adapted vaccination protocol, eventually leading to rabies elimination in 2006 and declaration of Germany as officially free of terrestrial rabies in 2008 (Selhorst et al., 2012). The RhinelandPalatinate outbreak was the first after a 7-year period of absence and thus appearing in a completely unprotected fox population. As a direct result, our analysis concentrates on a purely non-vaccinated area strictly before 2005 and highlights the effects resulting from outbreak defence strategies based on strict vaccination protocols.

Until now, a limited number of data analyses have been carried out and they have been based on cellular automata (CA), where time and space are treated as discrete. Formally, CA assumes a finite or denumerable set of homogeneous, simple units (cells) and a finite set of states evolving in parallel at discrete time steps (Eisinger and Thulke, 2008). For applications of CA in animal disease modelling see Thulke et al. (1999) and the literature cited therein.

This paper presents an alternative approach within the continuous spatio-temporal domain compared to CA. Besides the spatio-temporal analysis, we calculated statistics related to the spatial as well as temporal domain separately. The analysis was carried out using $\mathrm{R}$ and the stpp-package (R Core Team, 2014; Gabriel et al., 2013). 


$$
g\left((s, t),\left(s^{\prime}, t^{\prime}\right)\right)=\frac{\lambda_{2}\left((s, t),\left(s^{\prime}, t^{\prime}\right)\right)}{\lambda\left((s, t) \lambda\left(s^{\prime}, t^{\prime}\right)\right)}
$$

eq. 6

\section{Data}

The datasets used were provided by the Federal Research Institute for Animal Health (Riems, Germany) based on reported cases of the responsible veterinary authorities. A 14-month subsample was taken containing cases recorded from January 1, 2005 to February 28, 2006. Within this period, 36 events related to 25 dates were observed within time $\mathrm{T}=[14.01 .2005,03.02 .2006]$ and area $\mathrm{S}=[(372,890.9 ; 464,352.7)]$, $(5,424,623 ; 5,548,544)]$ consisting of 34 foxes and 1 horse as well as 1 roe-deer incident to fox rabies.

\section{Characteristics of spatio-temporal point processes}

Point process data is most often described using first- and second-order intensity functions as well as distance related measures. For a given random countable set of $k$ events $\left\{\left(s_{i}, t_{i},\right), i=1, \ldots\right.$ $, k\}$ we can define the intensity $l(s, t)$ of the process as:

$$
\lambda(s, t)=\lim _{\mid \begin{array}{l}
d s \rightarrow 0 \\
d t \rightarrow 0
\end{array}} \mid \frac{\mathbb{E}[N(d s, d t)]}{|d s||d t|}
$$

where $d s$ refers to an infinitesimal neighbourhood; $d t$ an infinitesimal interval in time; and $\mathrm{N}(\mathrm{ds}, \mathrm{dt})$ the number of events in $d s d t$. Hence $\lambda$ $(s, t)$ represents the expected (mean) number of events per unit area per unit time which can be estimated from empirical data as:

$$
\widehat{\lambda}=\frac{n}{|S \times T|}
$$

If the first-order intensity is invariant under translation such that $\lambda$ $(s, t)=\lambda$ holds for all pairs $(s, t)$ in $S \times T$, we call the stochastic spatiotemporal point process to be first-order or strongly stationary (homogeneous). Besides focusing on the mean number of events per unit area per unit time, we can derive the second-order properties, which are related to the variance of events per unit area per unit time as follows. Setting $t, t^{\prime} \in T$ and $s, s^{\prime} \in S$, where $t \neq t^{\prime}, s \neq s^{\prime}$ we can define the second-order intensity function as:

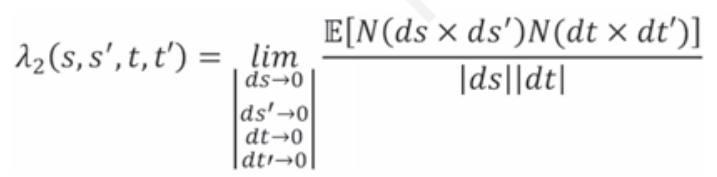

In addition, conditional on t,s' the second-order conditional intensity is given as:

$$
\lambda_{c}\left(s, t^{\prime} \mid s^{\prime}, t\right)=\frac{\lambda_{2}\left((s, t),\left(s^{\prime}, t^{\prime}\right)\right)}{\left.\lambda\left(s^{\prime}, t\right)\right)}
$$

Besides the already described intensity functions, the covariance density $\gamma\left((s, t),\left(s^{\prime}, t^{\prime}\right)\right)$ and the point pair correlation function $g$ $\left((s, t),\left(s^{\prime}, t^{\prime}\right)\right)$ as distance related measures can be used to describe second order properties. These are defined as:

$$
\gamma\left((s, t),\left(s^{\prime}, t^{\prime}\right)\right)=\lambda_{2}\left((s, t),\left(s^{\prime}, t^{\prime}\right)\right)-\lambda\left((s, t) \lambda\left(s^{\prime}, t^{\prime}\right)\right)
$$

respectively

Obviously, both measures relate the second-order intensity to a product of the corresponding first-order properties.

Based on the distribution of $\lambda_{2}\left(s, s^{\prime}, t, t^{\prime}\right)$ over the observation window, we can additionally gain insights into second-order stationarity. Thus, a spatio-temporal point process is said to be second-order stationary if, and only if $\lambda_{2}\left(s, s^{\prime}, t, t^{\prime}\right)=\lambda_{2}\left(s-s^{\prime}, t-t^{\prime}\right)$ is satisfied. Besides, we call a process isotrophic if the second-order intensity is invariant under rotation such that it can be rewritten as $\lambda_{2}\left(\| s-s^{\prime}||,\left|t-t^{\prime}\right|\right)$. Alternatively, setting $u=\| s-s^{\prime}||$ and $v=\left|t-t^{\prime}\right|$, a point is called secondorder reweighted stationary if its intensity function is bounded away from zero and its pair correlation function only depends on $u$ and $v$ (Diggle et al., 1995; Gabriel and Diggle, 2009).

\section{Spatio-temporal separability}

Separability embodies one possibility to reduce the complexity of spatio-temporal modelling by using separable temporal and spatial processes. Let $\lambda_{\mathrm{S}}(\mathrm{t})$ and $\lambda_{\mathrm{T}}(\mathrm{s})$ be the marginal spatial and temporal intensities, respectively. Then, a spatio-temporal point process is said to be first order separable if and only if $\lambda(s, t)=\lambda_{S}$ (t) $\lambda_{T}(s)$. Additionally, the process is said to be second-order separable if, and only if $g\left((s, t),\left(s^{\prime}, t^{\prime}\right)\right)=\mathrm{g}\left(\| s-s^{\prime} \mid\right) \mathrm{g}\left(\left|t-t^{\prime}\right|\right)$.

\section{Spatio-temporal K-function}

An alternative possibility to describe the second-order properties of a spatio-temporal process can be achieved by using Ripley's K-function encoding the probability of an arbitrary event within a certain distance in space. Assuming stationarity and isotrophy, this immediately leads to the following expression:

$$
K(u, v)=\lambda^{-1} \mathbb{E}\left[N^{0}\left(\left\|s-s^{\prime}\right\|,\left|t-t^{\prime}\right|\right)\right]
$$

where $\mathrm{N}^{0}$ refers to the number of further events within $\left\|s-s^{\prime}\right\| \in \mathrm{S}$ It $\mathrm{t}^{\prime} \mid \in \mathrm{T}$. Thereby, $\| \mathbf{s}-\mathbf{s}^{\prime}|| 2\left|\mathrm{t}-\mathrm{t}^{\prime}\right| \boldsymbol{\tau}>\mathrm{K}(u, v)$ indicates regularity, whereas aggregation follows if $\left\|\mathbf{s}-\mathrm{s}^{\prime}\right\| 2\left|\mathrm{t}-\mathrm{t}^{\prime}\right| \pi>\mathrm{K}(u, v)$ (Gabriel and Diggle, 2009). Alternatively, we used an unbiased version of Ripley's K-function for inhomogeneous spatio-temporal processes as introduced by Gabriel (2014):

$$
K(u, v)=\sum_{j \notin i} w_{i j}^{-1} \frac{1}{\lambda\left(x_{i}\right) \lambda\left(x_{j}\right)} \mathbb{I}_{\left\|s_{i}-s_{j}\right\| \leq u,\left|t_{i}-t_{j}\right| \leq v} \quad \text { eq. } 8
$$
where $\mathrm{w}_{\mathrm{ij}}{ }^{1}$ is the inverse of an edge correction factor, $\square$ is an indicator function, $u$ is $\left\|\mathbf{s}-\mathbf{s}^{\prime}\right\|$ and $v$ is It - $\mathbf{t}^{\prime}$.

\section{Results}

\section{Temporal evolution}

Restricted to the evolution of the process in time, we found a rapid increase within the first two months within the timeframe considered. The corresponding epidemic curve is shown in Figure 1.

As visualized by the epidemic curve, approximately $80 \%$ of all infections occurred in the first three months. Additionally, treating animal type as mark of a purely spatial process shows a clear centred spatial pattern at the eastern border of the observation window, which corresponds to the river Rhine as shown in Figure 2. Additionally to this plot, we investigated the spatial distribution by calculating the so called 


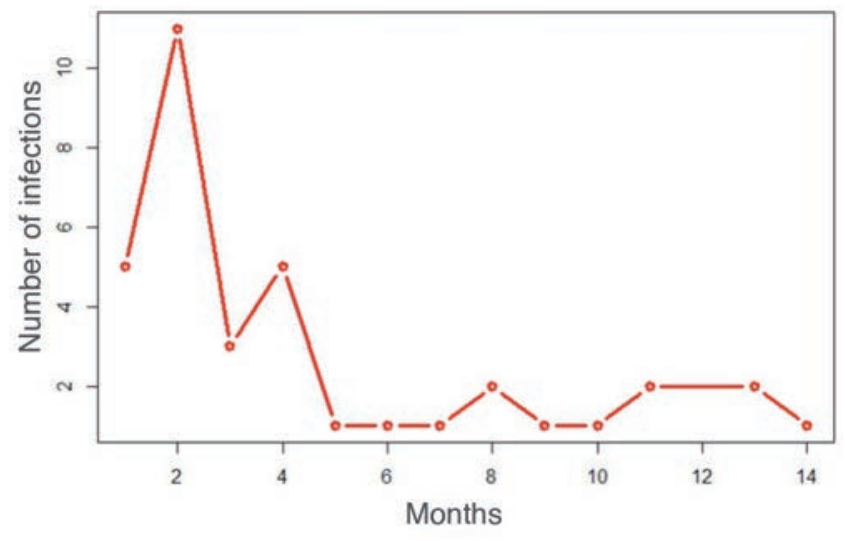

Figure 1. Rabies incidence evolution in the temporal domain.

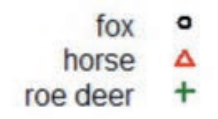

$\circ$

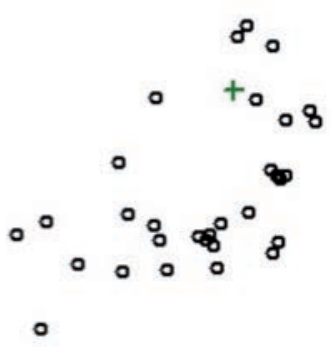

Figure 2. Spatial point process with animal as mark.

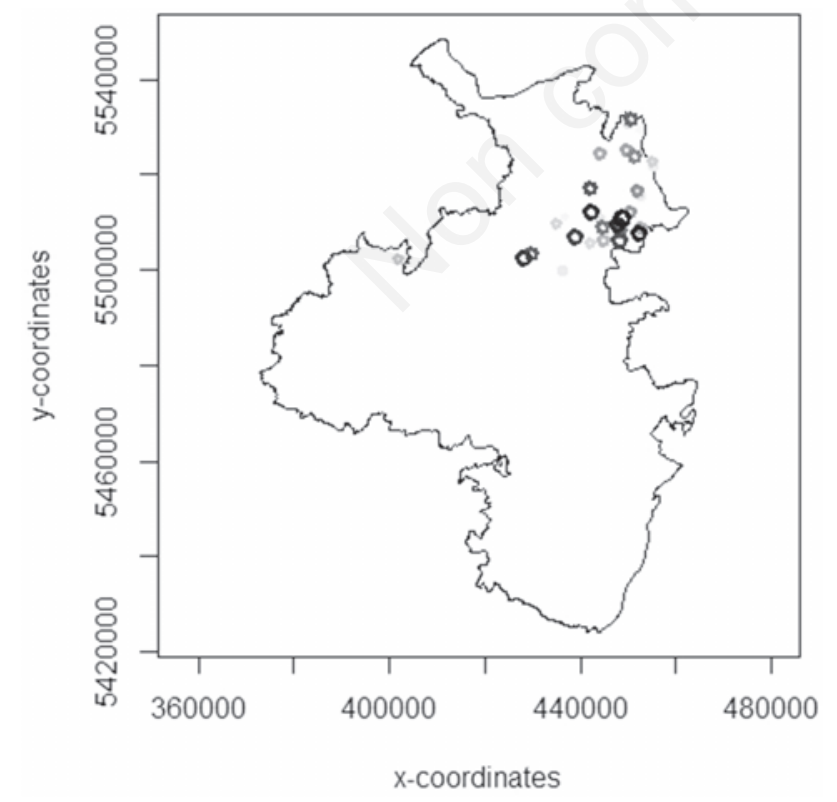

Figure 3. Temporally marked point process. Different colour shades correspond to the evolving time from light grey to black: the most current events are shown as completely black. The polygonal borders correspond to the analyzed districts belonging to the Rhineland-Palatinate State.

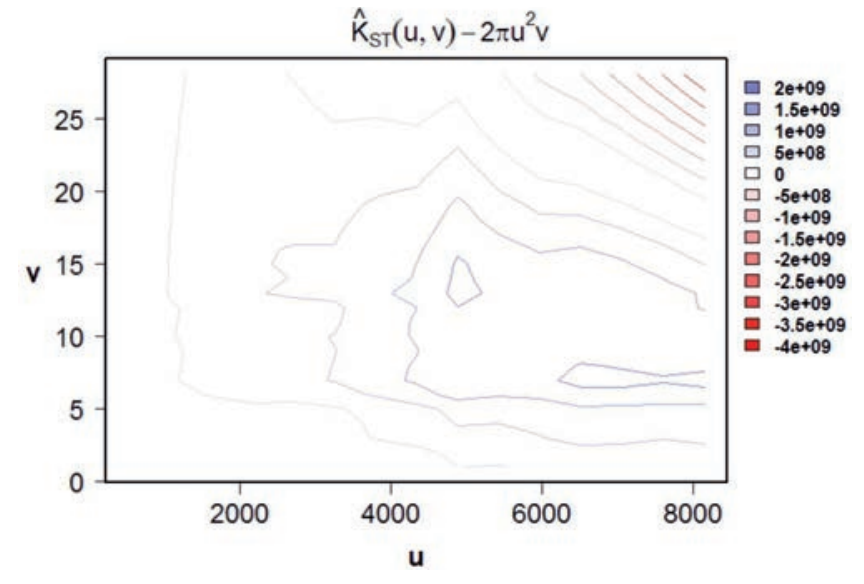

Figure 4. The spatio-temporal inhomogeneous K-function.

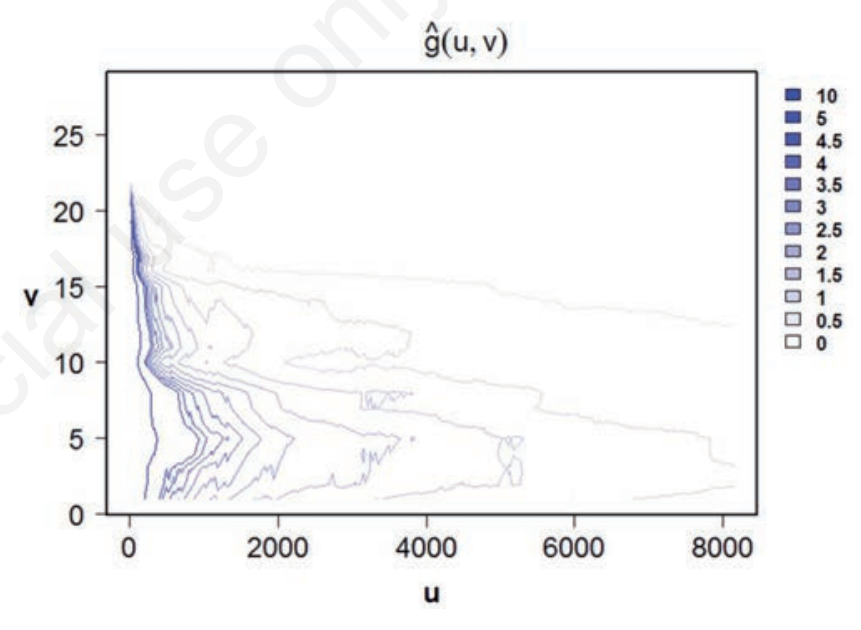

Figure 5. The space-time inhomogeneous pair correlation function.
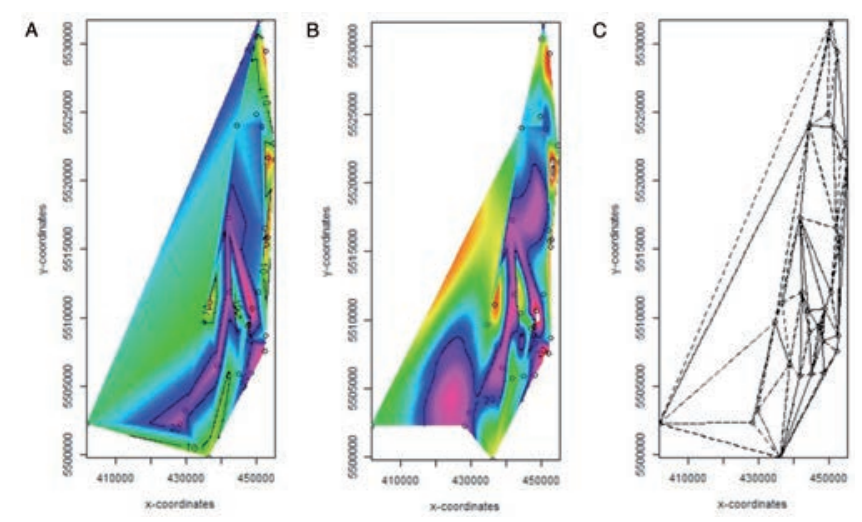

Figure 6. Mean time per area smoothing 2005 (A) to 2006 (B). C) displays Delaunay triangulation algorithm related to the spatial location. 
quadrate counting. Here, we divided the observation window into similar spaced sub-regions based on a regular grid and counted the number of observations falling into each quadrate. Under homogeneity, the distribution of counts would appear similar and no clear pattern would emerge.

\section{Spatio-temporal processes}

As discussed in Diggle (2014) and Schoenberg et al. (2006) a spatiotemporal point process can be investigated as a spatially or temporally marked. Therefore, we treated the spatio-temporal point process as temporally marked aiming to identify a certain pattern in time and space. The resulting plot is shown in Figure 3.

Again, the spatial pattern displayed in Figure 2 is found in Figure 3 showing a clear centric structure in the very upper right corner. In order to specify the observed pattern in more detail we calculated the corresponding $\mathrm{K}$ function and pair-correlation function. To investigate if spatio-temporal clustering or regularity of the observed point pattern exists we computed $K(u, v)-2 \pi u 2 v$. Thus, negative values indicate $K$ $(u, v)<2 \pi u 2 v$. The resulting plot is shown in Figure 4, where the $v=$ Itt'l distances are displayed on the y axis while the $u=\| s$ - s'll distances are shown on the $\mathrm{x}$ axis. Obviously, inspecting the contour lines visualizes a huge variation of $\mathrm{K}(u, v)-2 \pi u 2 v$. A clearly centroid structure emerged where blue colours relate to aggregation and red colours to regularity. Thus, this plot emphasized a highly aggregated point pattern within $u=[1500 ; 8000]$ and $v=[5 ; 25]$. In contrast, we observed regularity only in the very upper right corner of Figure 4.

A similar interpretation is obtained with respect to the pair correlation function as plotted in Figure 5, where we again observed a highlevel pair correlation within small spatial and temporal distances. This relation slightly decreased from bottom to top and left to right and vanished completely when inspecting the upper boundaries of $u$ and $v$.

We calculated also a Kernel density estimate as described in Akima $(1978,1996)$ based on bilinear (left panel) or bi-cubic spline (middle panel) smoothing as shown in Figure 6. Here, the bilinear smoothing was computed using a Delaunay triangulation algorithm related to the spatial location as displayed in the right panel of Figure 6. Neighboring locations resulting from triangular tessellation in space were used to determine the coefficients of the interpolation polynomial indicating the mean intensity in time. Here, the yellow shaded red colors correspond to high mean times, while blue and green colours encode lower mean time values. Both densities showed a similar temporal intensity pattern. The red highlighted areas of both contour plots completely intersected with the geographical location of large cities in the Rhineland-Palatinate State.

\section{Discussion}

The occurrence of fox rabies in Rhineland-Palatinate has been shown to be inhomogeneous in time and in space. We discovered a strongly aggregated pattern strictly limited to one area within the observation window. Nonetheless, limitation towards number of reported cases per area unit per time have to be taken into account when evaluating the findings. Also, the analysis was based on a dataset obtained within a pseudo-experimental environment due to the fact that the outbreak occurred in a non-vaccinated geographical area.

Before Germany achieved the rabies-free status, the control activities were often challenged and some areas put at risk during severe setbacks in the final phase of rabies elimination. In RhinelandPalatinate, fox rabies re-emerged in 2005 after a long period of absence (Muller et al., 2005, 2012). The rapid westward spread prompted veteri- nary authorities to react swiftly applying a new unproven vaccination strategy developed after re-infection of Carinthia, Austria in 2002 (Vogl, 2002). Application of this strategy proved to be successful in terrestrial rabies eradication.

The strategy used large-scale, aerial application of baits in short intervals in order to rapidly raise the vaccination rate. Nevertheless, it was observed that this objective could not be readily achieved in urban areas (Selhorst et al., 2012). The reasons were a high urban fox population density and the impossibility of large-scale aerial application of the vaccine-baits in urban and suburban areas due to safety regulations (Harris and Smith, 1987; Hegglin et al., 2004). Consequently, the rabies retracted to urban areas and could only be controlled after considerable effort including distributing bait by hand.

\section{Conclusions}

The analysis presented in this paper represents the first approach to fox rabies in Germany as a spatio-temporal point process. Thus, instead of separately analyzing the spatial and the temporal components, we rigorously modelled the disease evolution in space and time. Different to $\mathrm{CA}$ we treated time and space as continuous within a bounded observation window. It is noteworthy that the proposed smoothers identified urban areas as high-risk for the occurrence of fox rabies. This confirms the existing theories for the control of rabies and draws again the focus of attention on the development of efficient strategies in urban areas.

\section{References}

Akima H, 1978. A method of bivariate interpolation and smooth surface fitting for irregularly distributed data points. ACM T Math Software 4:148-64.

Akima H, 1996. Algorithm 761: scattered-data surface fitting that has the accuracy of a cubic polynomial. ACM T Math Software 22:36271.

Cressie NAC, Wikle CK, 2011. Statistics for spatio-temporal data. John Wiley and Sons, Hoboken, NJ, USA.

Diggle PJ, 2014. Statistical analysis 366 of spatial and spatio-temporal point patterns. 3rd rev. ed. Chapman and Hall, London, UK.

Diggle PJ, Chedwynd A, Haggkvist R, Morris S, 1995. Second-order analysis of space-time clustering. Stat Methods Med Res 4:124-36.

Eisinger D, Thulke HH, 2008. Spatial pattern formation facilitates eradication of infectious diseases. J Appl Ecol 45:415-23.

Freuling C, Hampson K, Selhorst T, Schröder R, Meslin FX, Mettenleiter TC, Muller T, 2013. The elimination of fox rabies from Europe: determinants of success and lessons for the future. Philos T Roy Soc B 368:20120142.

Gabriel E, 2014. Estimating second-order characteristics of inhomogeneous spatio-temporal point processes: influence of edge correction methods and intensity estimates. Methodol Comput Appl 16:1.

Gabriel E, Diggle PJ, 2009. Second-order analysis of inhomogeneous spatio-temporal point process data. Stat Neerl 63:43-51.

Gabriel E, Rowlingson B, Diggle PJ, 2013. Stpp: an R package for plotting, simulating and analyzing spatio-temporal point patterns. J Stat Softw 53:1-29.

Harris S, Smith GC, 1987. Demography of two urban fox (Vulpes vulpes) populations. J Appl Ecol 24:75-86.

Hegglin D, Bontadina F, Gloor S, Romer J, Muller U, Breitenmoser U, Deplazes P, 2004. Baiting red foxes in an urban area: a camera trap 
study. J Wildlife Manage 68:1010-7.

Muller T, Bätza HJ, Freuling C, Kliemt A, Kliemt J, Heuser R, Schluter $\mathrm{H}$, Selhorst T, Vos A, Mettenleiter T, 2012. Elimination of terrestrial rabies in Germany using oral vaccination of foxes. Berl Munch Tierarztl 125:178-90.

Muller T, Selhorst T, Potzsch C, 2005. Fox rabies in Germany - an update. Euro Surveill 10:229-31.

R Core Team, 2014. R: a language and 414 environment for statistical computing. R Foundation for Statistical Computing, Vienna, Austria.

Schoenberg FP, Brillinger DR, Guttorp P, 2006. Point processes, spatialtemporal, encyclopedia of environmetrics. John Wiley and Sons, Hoboken, NJ, USA.

Selhorst T, Freuling CM, Blicke J, Vos A, Larres G, Zimmer K, Fröhlich T, Bätza HJ, Muller TF, 2012. Short term interval baiting to combat the re-emergence of fox rabies in Rhineland Palatinate (Germany) in 2005. Berl Munch Tierarztl 125:191-196.

Thulke HH, Grimm V, Muller MS, Staubach C, Tischendorf L, Wissel C, Jeltsch F, 1999. From pattern to practice: a scaling-down strategy for spatially explicit modelling illustrated by the spread and control of rabies. Ecol Model 117:179-202.

Vogl D, 2002. The reinfection of rabies of Karnten (Carinthia) in Austria - the run of events. Rabies Bull Eur 26:10-1.

World Health Organization, 2013. Expert 439 consultation on rabies. Second report. WHO Tech Rep Ser 982:1-150. 